\title{
BMJ Open Quality Bundle approach used to achieve zero central line-associated bloodstream infections in an adult coronary intensive care unit
}

\author{
Poonam Gupta (D) , ${ }^{1}$ Mincy Thomas, ${ }^{2}$ Ashfaq Patel, ${ }^{3}$ Reeba George, ${ }^{2}$ \\ Leena Mathews, ${ }^{2}$ Seenu Alex, ${ }^{2}$ Siji John, ${ }^{2}$ Cherlyn Simbulan, ${ }^{4}$ Ma Leni Garcia, ${ }^{4}$ \\ Sara Al-Balushi, ${ }^{5}$ Mawahib El Hassan ${ }^{3}$
}

To cite: Gupta P, Thomas M,

Patel A, et al. Bundle approach used to achieve zero central line-associated bloodstream infections in an adult coronary intensive care unit. BMJ Open Quality 2021;10:e001200. doi:10.1136/ bmjoq-2020-001200

Received 9 September 2020 Revised 29 December 2020 Accepted 7 February 2021

Check for updates

(C) Author(s) (or their employer(s)) 2021. Re-use permitted under CC BY-NC. No commercial re-use. See rights and permissions. Published by BMJ.

${ }^{1}$ Quality Improvement Department, Hamad Medical Corporation, Doha, Qatar

${ }^{2}$ Nursing Department, Hamad Medical Corporation, Doha,

Qatar

${ }^{3}$ Consultant Cardiology, Hamad Medical Corporation, Doha,

Qatar

${ }^{4}$ Infection Control Department, Hamad Medical Corporation,

Doha, Qatar

${ }^{5}$ Pharmacy Department, Hamad Medical Corporation, Doha, Qatar

Correspondence to Dr Poonam Gupta;

PGupta1@hamad.qa

\section{ABSTRACT}

Background Central venous catheterisation is commonly used in critical patients in intensive care units (ICU). It may cause complications and attribute to increase mortality and morbidity. At coronary ICU (CICU) of cardiac hospital, central line-associated bloodstream infection (CLABSI) rate was 2.82/1000 central line days in 2015 and 3.11/1000 central line days in 2016. Working in collaboration with Institute for Healthcare Improvement (IHI), we implemented evidence-based practices in the form of bundles in with the aim of eliminating CLABSI in CICU.

Methods In collaboration with IHI, we worked on this initiative as multidisciplinary team and tested several changes. CLABSI prevention bundles were tested and implemented, single kit for line insertion, simulationbased training for line insertions, standardised and realtime bundle monitoring by direct observations are key interventions tested. We used model for improvement and changes were tested using small Plan-Do-Study-Act cycles. Surveillance methods and CLABSI definition used according to National Healthcare Safety Network.

Results The CLABSI rate per 1000 patient-days dropped from 3.1 per 1000 device-days to 0.4 per 1000 devicedays. We achieved 757 days free of CLABSI in the unit till December 2018 when a single case happened. After that we achieved 602 free days till July 2020 and still counting. Conclusions Implementation of evidence-based CLABSI prevention bundle and process monitoring by direct observation led to significant and subsequently sustained improvement in reducing CLABSI rate in adult CICU.

\section{INTRODUCTION}

Healthcare-associated infections are a leading cause of morbidity and mortality. ${ }^{1}$ Central line-associated bloodstream infections (CLABSIs) are serious infections that can lead to increases in the length of hospital stays, case costs and mortality and morbidity. ${ }^{2}$ CLABSIs are a global healthcare problem despite the availability of guidelines, education and equipment to manage it. Most of these infections are preventable if evidencebased practices (including surveillance), insertion and maintenance guidelines are followed. - $^{3-5}$

CLABSI is defined as a laboratoryconfirmed BSI that occurs when an eligible BSI organism is identified and a central line is present when the BSI is laboratory confirmed. The specific definitions related to BSI/CLABSI surveillance are listed in table 1 .

CLABSI causes significant increases in morbidity, mortality and healthcare costs. ${ }^{67}$ Nosocomial BSIs may prolong hospitalisation by an average of 7 days, and the estimated cost per BSI is between US $\$ 3700$ and US $\$ 29000 .^{8}$ There is a considerable amount of literature demonstrating that best practices, protocols, checklists and establishing a culture of patient safety can reduce the incidence of CLABSI, or eliminate it entirely. Moreover, it has been shown that the effective implementation of central line insertion and maintenance bundles can reduce the incidence of CLABSI in intensive care units (ICUs) and in non-ICU settings. ${ }^{9-11}$ Bundle is sets of evidence-based measures that, when implemented together, have been shown to produce better outcomes and have a greater impact than the implementation of individual measures. In addition, the bundles also help to create reliable and consistent care systems in hospital settings, as they are simple (3-5 elements), concise and clear to interpret and implement. ${ }^{12}$

In 2015 and 2016, the coronary ICU (CICU) at our cardiac hospital observed high CLABSI rates. The total number of reported cases was 4 out of 1416 central line days in 2015 (a rate of 2.82/1000 central line days) and 6 cases out of 1931 central line days in 2016 (a rate of 3.11/1000 central line days). The CLABSI rate at the CICU was, during this period, higher than the National Healthcare Safety Network's (NHSN's) 50th percentile benchmark of $0.8 .^{13}$ Therefore, a thorough 
Table 1 Operational definitions specific to BSI/CLABSI surveillance $^{13}$

Primary BSI A laboratory-confirmed bloodstream infection that is not secondary to an infection at another body site

$\begin{array}{ll}\text { Secondary BSI } & \text { A BSI that is thought to be seeded from a site-specific } \\ \text { infection at another body site }\end{array}$

Central line $(\mathrm{CL})$ : An intravascular catheter that terminates at or close to the heart, or in one of the great vessels used for infusion, the withdrawal of blood, or haemodynamic monitoring. The following great vessels should be considered when making determinations regarding CLABSI events and counting CL device days:

- Aorta

- Pulmonary artery

- Superior vena cava

- Inferior vena cava

- Brachiocephalic veins

- Internal jugular veins

- Subclavian veins

- External iliac veins

- Common iliac veins

- Femoral veins

$\begin{array}{ll}\text { Eligible CL } & \text { A CL that has been in place for more than two } \\ & \text { consecutive calendar days (on or after CL day 3), } \\ \text { following first access to the central line during the } \\ \text { current admission. Such lines are eligible for CLABSI } \\ \text { events and remain eligible for CLABSI events until } \\ \text { the day after its removal from the body or patient } \\ \text { discharge, whichever comes first. }\end{array}$

BSI, bloodstream infection; CLABSI, central line-associated bloodstream infection; NHSN, National Healthcare Safety Network.

analysis was undertaken by our improvement task force and interventions were focused on the CICU, where patients were acutely ill and suffered from multiple co-morbidities. Subsequent to performing a root-cause analysis, we concluded that there was a significant lack of understanding the importance of central line maintenance measures. As there is some evidence indicating that bundled educational and behavioural interventions are effective in reducing CLABSI incidence, ${ }^{14-20}$ our assumption was that the implementation of a central line maintenance bundle approach would result in CLABSI reductions.

There were cases of CLABSI in the unit at different intervals and no standardised process was in place. The purpose of this study was to identify the causes, implement and evaluate a central line care insertion and maintenance bundle designed to optimise central line infection prevention practices and reduce CLABSI in adult, cardiac ICU settings. In addition, we sought to improve compliance for central line maintenance bundles. Moreover, we aimed achieve 365 CLABSI-free days in the CICU by 31 December 2018 by following evidence-based practices using a multidisciplinary team approach.

\section{METHODS \\ Context}

Our tertiary facility has a 20-bed CICU that serves cardiac patients. In September 2016 a Stop CLABSI Project was undertaken as a part of a Best Care Always initiative. To build improvement capability in front-line teams, the Hamad Medical initiated collaboration with the Institute for Healthcare Improvement (IHI) as part of the Best Care Always initiative. Capability building in front-line staff had previously led to a number of quality improvements (QI) initiatives, and teams had successfully performed other QI collaborations. ${ }^{21}$

\section{CLABSI prevention bundle}

The CLABSI prevention bundles (box 1) are a collection of care bundles ${ }^{22-25}$ and details are listed in box 1 .

These bundles are all evidence-based practices with ample literature supporting them. ${ }^{26}$ In preparation for the introduction of the bundles in practice, all bedside nurses were educated on the bundle elements. Each element of the bundles required several tests of change. Compliance with the elements was ensured using an audit form.

\section{Interventions}

Using the IHI's collaborative model, this initiative comprised a multidisciplinary team that included nurses, physicians, infection-control practitioners and QI professionals. The team undertook comprehensive case reviews for every CLABSI incidence that occurred in the CICU to identify and implement best practices with the aim of reducing CLABSI rates in the adult, cardiac ICU setting.

After brainstorming and performing a Pareto analysis, we concluded that there were practice gaps concerning compliance for both the insertion and maintenance bundles. This included a lack of awareness regarding the implementation and monitoring of each component of the bundles. We used the Model for Improvement, which is composed of three components, to structure and guide improvements. ${ }^{27}$ These components were: set an aim statement, define measures and select small changes to test. We use small Plan-Do-Study-Act (PDSA) cycles to 
Box 1 Central line-associated bloodstream infection care bundles

\section{Central line insertion bundle \\ Hand hygiene \\ - Wash hands or use an alcohol-based, waterless hand cleaner when caring for central lines: \\ Before and after palpating the catheter insertion site. \\ Before and after inserting, replacing, adjusting or dressing the site. During palpation of the insertion site and after application of anti- septic and only if full asepsis is maintained.}

Complete maximal barrier

- The operator inserting the central venous catheter should adhere to strict aseptic techniques and wear sterile gloves, gown, surgical cap and surgical mask.

\section{Chlorhexidine $2 \%$ skin antisepsis}

- Prepare skin with $2 \%$ chlorhexidine in $70 \%$ alcohol using swabs and a friction scrub for at least 30 secondss. Do not wipe or blow dry and allow to dry completely before skin puncture:

$30 \mathrm{~s}$ for a dry site.

2 min for a moist site (especially femoral).

\section{Optimal catheter site selection}

- In adult patients, there is some evidence that the subclavian site has a lower risk of catheter-related blood stream infections. However, there is usually more experience with the internal jugular site. The subclavian and internal jugulars are the preferred sites for infectioncontrol purposes.

- Use of sterile, single-use jelly.

- Use sterile, single-use jelly for ultrasound-guided insertions.

Central line maintenance bundle

\section{Hand hygiene}

- Practice hand hygiene at five moments ${ }^{33}$.

Before touching a patient.

Before clean/aseptic procedures.

After body-fluid exposure/risk.

After touching a patient.

After touching patient surroundings.

\section{Aseptic technique for accessing and changing needleless}

connectors

- Scrub the access port or hub immediately prior to each use with an appropriate antiseptic.

\section{Standardised tubing change}

- Intravenous medication administration tubing should be changed as per the recommendation in the local organisations policy.

\section{Daily review of catheter necessity}

- Daily review of line necessity during rounds so that the necessity of the lines can be determined and unnecessary lines removed.

test changes in ideas and successful results were implemented. All key stakeholders assessed the current state of the CLABSI prevention process bundles and their redesign. Based on previous analyses, several changes were tested using multiple PDSA cycles.

\section{PDSA 1: CLABSI bundle audit form}

A CLABSI bundle-checklist audit tool was developed and tested. It included insertion and maintenance elements and was first tested on one nurse and one patient during one shift. Once implemented, data were collected daily using the audit form and weekly compliance was calculated by a task force and communicated to the teams. The tool was modified based on feedback from nurses before it was fully adopted. Monthly data of the unit CLABSI rates were communicated to the staff through event calendars and monthly unit meetings.

\section{PDSA 2: hand hygiene}

PDSA 2: 1. Formal education on hand hygiene was conducted for all of the front-line staff working in CICU by an infection-control practitioner. The practice of hand hygiene at five moments was taught and monitored.

PDSA 2: 2. Compliance with the five moments of hand hygiene was monitored by secret observers. Daily compliance data, with individual names, was displayed on a unit board. Physicians and nurses with the highest compliance rates were acknowledged. This proved to be a successful method and the change was adopted.

PDSA 2: 3. Another test of change included handhygiene time, which was designated as 11 o'clock AM. An announcement was made through an ASCOM device to ensure the announcement went to all healthcare workers to remind them to perform this activity. The hand-hygiene campaign was periodically run in the department to reinforce the message.

PDSA 2: 4. Soap or alcohol-based hand-gel dispensers were prominently placed in or near patient rooms, and universal precautionary equipment such as gloves were made available near hand-sanitation equipment. Reminders were posted at the entry and exits to patient rooms.

\section{PDSA 3: all-inclusive central line kits}

Standard equipment for central line placements were stocked in a cart or kit to avoid any difficulties with finding necessary equipment to initiate the bundle elements. Teams prepared a list of all the consumable items required during central line insertions and, with the help of material management, they prepared kits. Theses kit consisted of all the required consumable items for a central line insertion in a single pack to minimise interruptions during the procedures. The pack was tested on one patient and one doctor during a single shift. Once feedback was collected, the change was adopted if it was shown to be successful.

\section{PDSA 4: maximal barrier precautions}

Proper use of personal protective equipment ${ }^{28} 29$ during the insertion and care of central venous catheters (CVCs) was monitored.

\section{PDSA 5: use of chlorhexidine skin antiseptics}

The use of chlorhexidine ${ }^{30}$ for skin antisepsis and proper technique was monitored. The disinfection of hubs and injection ports with alcohol cap port protectors was also performed. Chlorhexidine dressing and the daily use of chlorhexidine bath wipes for cleaning patients on CVCs was followed.

The evidence-based practice of pressing a sponge against the skin and applying chlorhexidine solution 
using a back-and-forth friction scrub for at least $30 \mathrm{~s}$ was also monitored.

We reinforced the practice of not wiping or blotting and, instead, allowed time for the antiseptic solution to completely dry before puncturing the site $(\sim 2 \mathrm{~min})$.

\section{PDSA 6: physician education on central line insertion}

The education and training of all physicians was undertaken through simulation techniques. Proper surgical hand scrubs, the principles of aseptic techniques during insertion, techniques for ultrasound-guided central line insertion and full maximum barriers during insertion were the focus of the educational sessions. In addition, emphasis was placed on preferred sites of choice (either the jugular or subclavian for central line insertion). Ultrasound guidance used to assess and detect the most suitable vein and replacement of CVCs over the guidewire was strongly discouraged.

\section{PDSA 7: bundle element compliance}

Turning the ventilator tubing away from the CVC site and regular oral suctioning was performed for patients with large amounts of secretions. The regular inspection of sites for any signs and symptoms of infection was performed, and daily assessments for the need of a CVC was implemented. Physicians also reassessed patients requiring long-term central lines and change them to peripherally inserted central catheter lines.

Reminders for how long a line had been in place were achieved by stating the line day (eg, 'line day 4') during rounds. Bundle compliance was measured using an all-ornothing approach and compliance data were shared with the staff.

\section{Data displays}

To keep momentum and create sense of urgency, data that included hand hygiene, bundle compliance and CLABSI rates were displayed on a unit quality board, which was easily accessible by the staff. Run charts and other information regarding the project were displayed in a central location.

\section{Intervention measures}

\section{Outcome measures}

The CLABSI rate was defined as the number of CLABSIs in the CICU/the number of central line days in the CICU $\times 1000$. Data collection was done via surveillance performed by infection-control practitioners. The central line days were calculated for the denominator. All patients in the CICU with central lines were included in the intervention.

\section{Process measures}

Each of the bundle elements was monitored for compliance. Compliance with each change was also measured (ie, per cent compliance with the bundle as a whole and for each of the bundle elements). Compliance was considered to have been achieved if all of the elements of a bundle were followed-even one component missing was considered to be zero compliance.

\section{Statistical analysis}

We analysed CLABSI rates for 19 months before implementing the interventions and 19 months following them. Data that departed from normality were tested using the Shapiro-Wilk test. Monthly rates of CLABSI were compared between the 19 months before and after the intervention using the Mann-Whitney $U$ test. The device utilisation ratio in the 19 months before and after the intervention was compared using the Student's independent t-test.

A two-tailed $\mathrm{p}<0.05$ was considered significant. Microsoft Excel 2016 and Stata/SE V.14.2 (StataCorp) was used for the analyses. The outcomes of the project were analysed using standard control chart rules, which detect statistically significant changes in outcomes over time. Compliance data were collected for every element as a process measure.

\section{RESULTS}

Following the implementation of the Stop CLABSI initiative, CLABSI was eliminated from the CICU for 757 days. There was a single case in December 2018, after which there was 602 days where the CICU was free of CLABSI.

Monthly rates of CLABSI during the 19 months after the intervention were significantly lower when compared with the 19 months before the intervention $(p=0.0495)$. The device utilisation ratio during the 19 months before and after the intervention was not significantly different $(p=0.0772)$. The annual count of CLABSI cases was reduced from 4 in 2015 and 6 in 2016 to 0 in 2017.

The CLABSI rate per 1000 patient-days dropped from 3.1 per 1000 device-days to 0.4 per 1000 device-days, which is below the NHSN benchmark of 0.8 (figure 1; quarterly data are displayed in figure 2). The run chart displayed in figure 2 is annotated to illustrate the implementation of some of the changes that were tested. It appeared that attention to the CLABSI prevention bundle had the largest effect. There was also a substantial improvement in bundle compliance, which increased from $64 \%$ to $100 \%$, an improvement that was sustained for more than 3 years (figure 3 ). There was no significant difference in the device utilisation ratio (figure 4).

\section{DISCUSSION}

During the Stop CLABSI initiative, we achieved considerable improvements in the CICU, as the implementation of the maintenance bundle of preventive measures resulted in the elimination of CLABSI from the unit. The number of CLABSI cases reported in 2015 and 2016 was 4 and 6, respectively, which was reduced to 0 in 2017, 2018 and 2019-with a single case in December 2018. The CLABSI rate per 1000 patient-days dropped from 3.1 per 1000 device-days to 0.4 per 1000 device-days. The two-tailed 


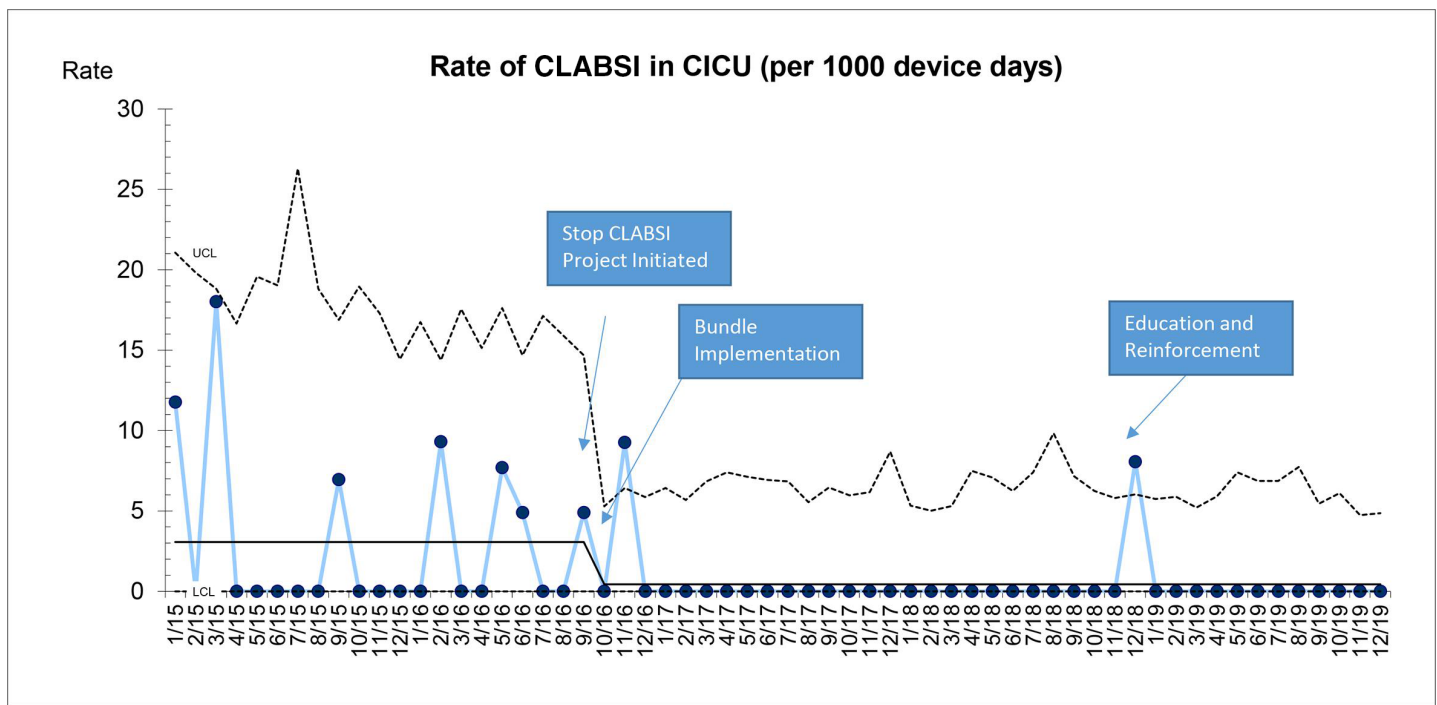

Figure 1 Monthly CLABSI rate in CICU Per 1000 device days. CICU, coronary intensive care unit; CLABSI, central lineassociated bloodstream infection.

$\mathrm{p}<0.05$ was significant. Bundle compliance increased from $64 \%$ to $100 \%$ and was sustained.

Front-line teams were advised to always follow each bundle element for every patient. In addition, our approach helped us to develop and promote a patient safety culture and, ultimately, build a reliable care process in the system. The multidisciplinary approach allowed us to brainstorm different explanations for the higher rates of CLABSI in the unit and assess any deficiencies. It also allowed us to propose new solutions from a variety of perspectives and angles, including from nurses and physicians and infection-control practices. All of the interventions were planned and customised based on patient need, which helped us to reduce the rate of CLABSI and maintain the improvements over the long term.

In addition to the implementations of bundles, regular face-to-face meetings and educational sessions that included simulations as well as one-on-one and small classroom sessions proved to be vital during the initiative. From our results, we concluded that the bundle approach was effective in implementing change and improving outcomes by promoting teamwork, measuring compliance and providing feedback. ${ }^{31}$ In addition, effective care-bundle implementation required that measures be adapted to the local setting, appropriately followed, suitable to the patient-care culture, and monitored and evaluated to ensure compliance. ${ }^{32}$

We attempted to recognise staff with the highest handhygiene compliance, and they were shown appreciation for their participation through emails and recognition on notice boards. Additional measures to promote compliance with the guidelines such as weekly posters and additional staff meetings were enacted to reach $100 \%$ compliance. This is a regular practice in the unit to appreciate staff for their good work to encourage them.

\section{Strengths, limitations and lessons learnt}

There are many factors which made this initiative a success, including direct observation of catheter insertion-site care practices, regular monitoring of compliance with the

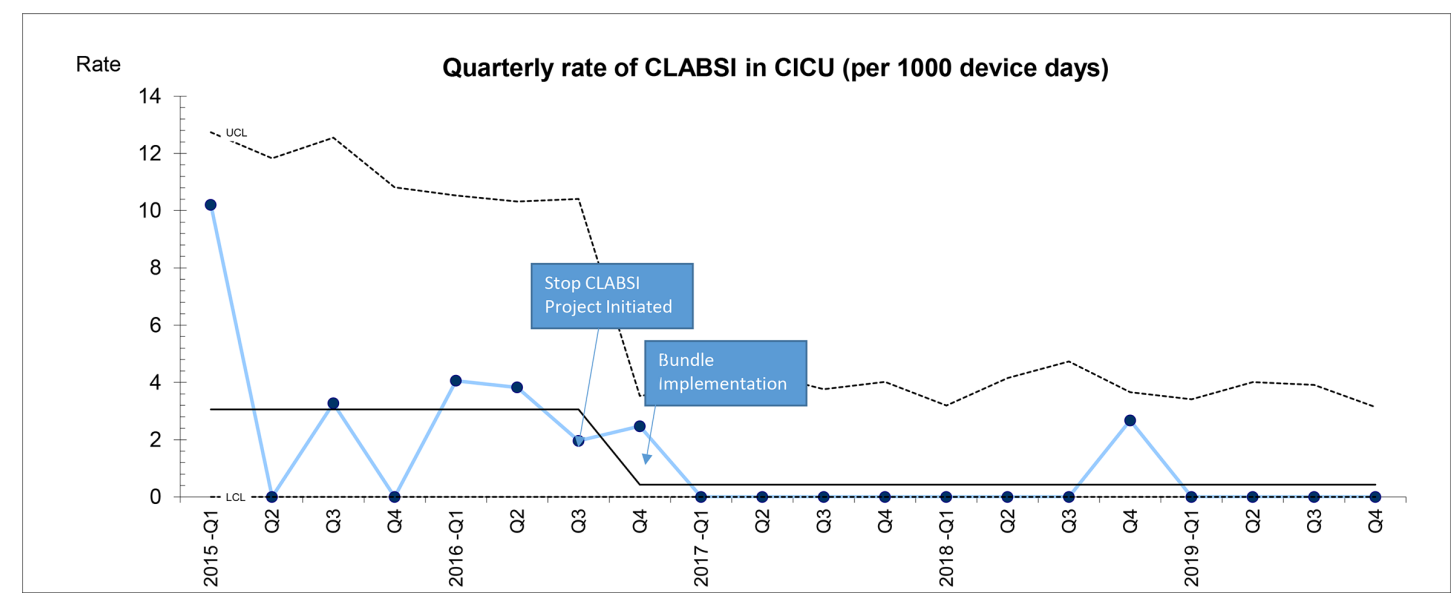

Figure 2 Quarterly rate of CLABSI in CICU per 1000 device days. CICU, coronary intensive care unit; CLABSI, central lineassociated bloodstream infection. 


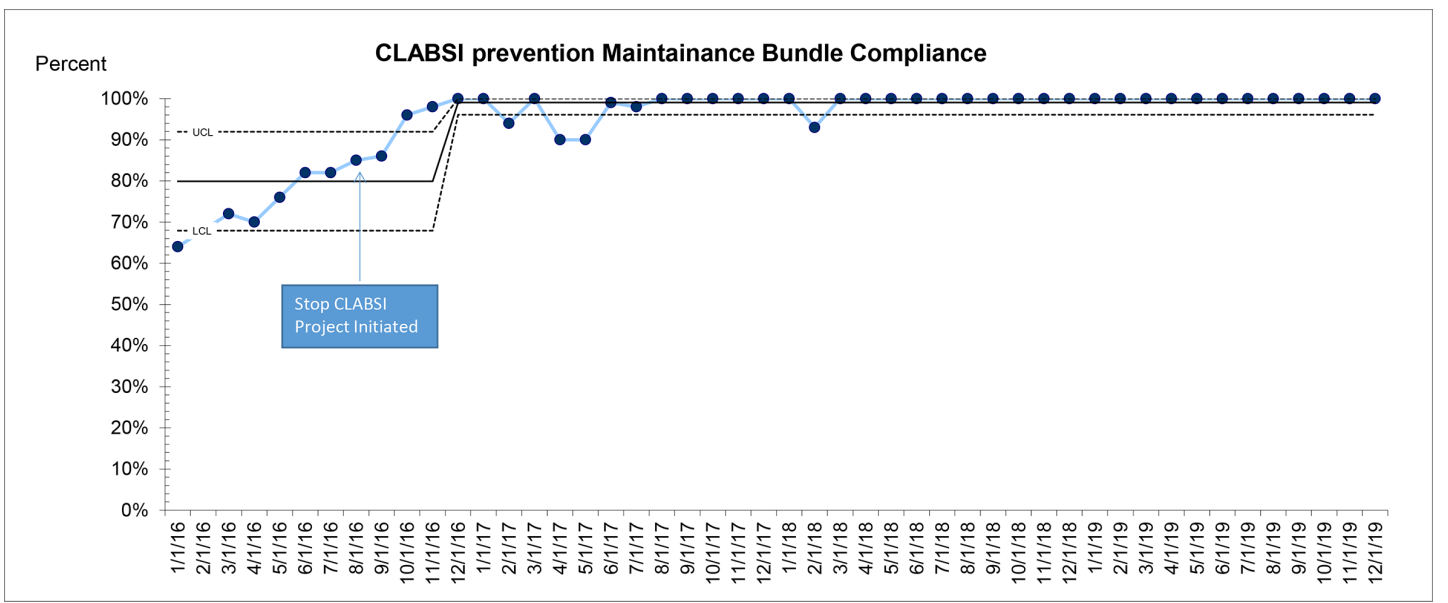

Figure 3 CLABSI prevention maintenance bundle compliance. CLABSI, central line-associated bloodstream infection.

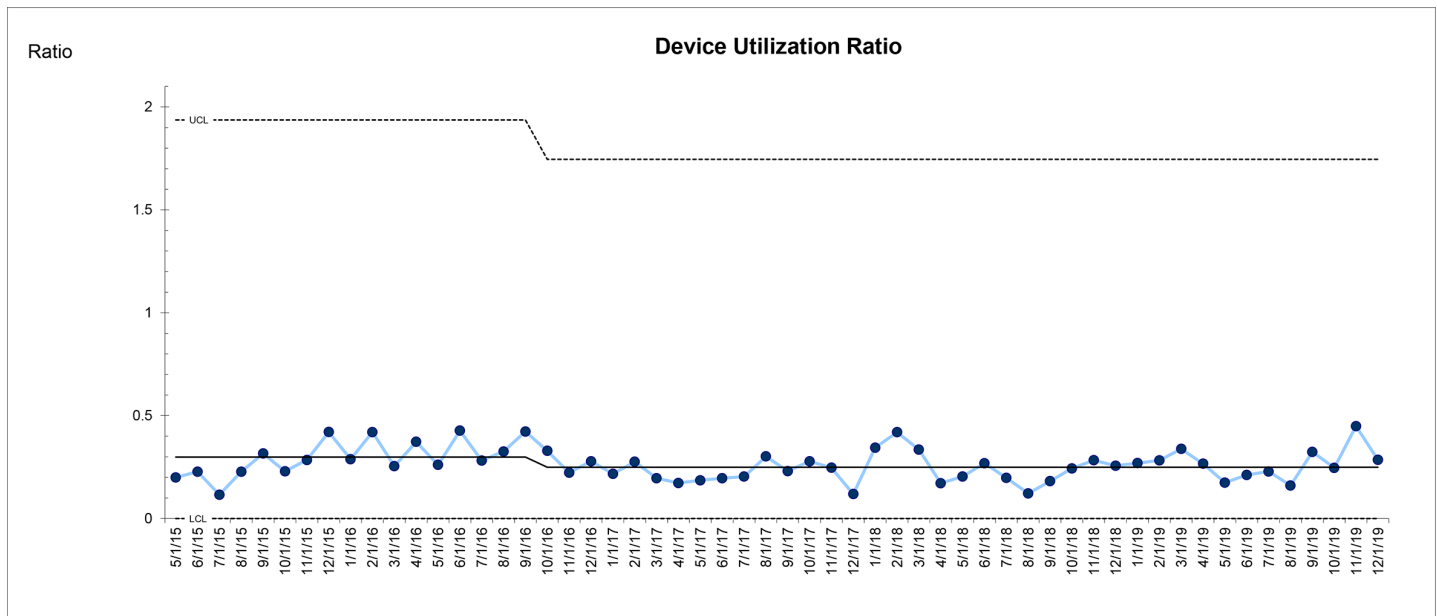

Figure 4 Device utilisation ratio.

bundles, use of ultrasound-guided insertions and training opportunity needs were identified and met for insertion and maintenance bundles. In addition, this work was led by front-line teams who were then able to apply the QI knowledge built during the Best Care Always initiative to their daily practices and for other initiatives in their clinical areas.

The main limitation of this study was the inability to differentiate which elements from the bundles had the strongest effect on case prevention and CLABSI incidence, as the bundles consisted of several elements that were implemented simultaneously. Likewise, as this study was not a randomised control trial and there was no control group, we were unable to assess effectiveness of each component of the bundle approach.

There were several lessons learnt during the course of this initiative. First, it is important to involve a multidisciplinary team that can affect both the decision-making process and interventions from the start. This helps with identifying key issues and in implementing an effective intervention to resolve them. Second, involving physicians for training and education purposes also played vital role, as well as the simulation exercises, which proved to be an effective learning experience.

\section{CONCLUSIONS}

CLABSIs are a serious but preventable healthcareassociated infection. Using a multifaceted approach that includes multidisciplinary teams that follow an evidencebased, bundled approach resulted in a significant reduction in CLABSI rates in an ICU setting. We will continue to assess new interventions/preventions as guided by infection-control teams and the recent literature to sustain the gains already achieved.

Acknowledgements We acknowledge Heart Hospital and Hamad Healthcare Quality Institute (HHQI) leadership, including Dr Nidal Asaad (Medical Director), Dr Awad Al Qahtani (Chairman Cardiology),Mr. Nasser Al Naimi (Deputy Chief Quality), Dr Salah Arafa (Director Performance Improvement), Mr lan McDonald (Executive Director Nusring), Mr Mohammad Al Zubi (Direcor of Nusring), Dr. Emad Bashier (Senior consultant, Laboratory) for their support for this initiative. In addition, we would like to extend our thanks to HHQI (Hamad Healthcare Quality Institute) and IHI for their support for Best Care Always initiative. Our special thanks to Dr Ezeddin Alataresh and frontline teams of CICU including physicians, nursing and allied health professionals for their continuous support to this work.

Contributors PG served as Quality Improvement advisor and prepared initial draft of this manuscript. MT led the study and supervised implementation of changes. $\mathrm{MH}$ and AP played key role in physician's education and interventions. CS and LG provided valuable guidance from infection control team. $R G, S A$ and $L M$ played vital role in planning and implementation of changes. SB assisted in statistical analysis. All authors contributed equally in the final approval of the manuscript. 
Funding There was no external funding for this project. The overall quality improvement effort 'Best Care Always' was entirely funded by the Hamad Medical Corporation

\section{Competing interests None declared.}

Patient and public involvement Patients and/or the public were not involved in the design, or conduct, or reporting, or dissemination plans of this research.

\section{Patient consent for publication Not required.}

Ethics approval The study was a hospital-based, infectious disease-control prevention initiative, IRB approval was not needed.

Provenance and peer review Not commissioned; externally peer reviewed.

Data availability statement All data relevant to the study are included in the article.

Open access This is an open access article distributed in accordance with the Creative Commons Attribution Non Commercial (CC BY-NC 4.0) license, which permits others to distribute, remix, adapt, build upon this work non-commercially, and license their derivative works on different terms, provided the original work is properly cited, appropriate credit is given, any changes made indicated, and the use is non-commercial. See: http://creativecommons.org/licenses/by-nc/4.0/.

ORCID iD

Poonam Gupta http://orcid.org/0000-0002-9654-244X

\section{REFERENCES}

1 The Joint Commission. Preventing central line-associated bloodstream infections; 2012.

2 Tacconelli E, Smith G, Hieke K, et al. Epidemiology, medical outcomes and costs of catheter-related bloodstream infections in intensive care units of four European countries: literature- and registry-based estimates. J Hosp Infect 2009;72:97-103.

3 Hallam C, Jackson T, Rajgopal A, et al. Establishing catheter-related bloodstream infection surveillance to drive improvement. $J$ Infect Prev 2018;19:160-6.

4 Aloush SM, Alsaraireh FA. Nurses' compliance with central line associated blood stream infection prevention guidelines. Saudi Med J 2018;39:273-9.

5 O'Grady NP, Alexander M, Burns LA, et al. "Guidelines for the Prevention of Intravascular Catheter-related Infections". Clinical Infectious Diseases 2011;52 (a:1087-99.

6 Burke JP. Infection control - a problem for patient safety. N Engl J Med 2003;348:651-6.

7 Mermel LA. Prevention of intravascular catheter-related infections. Ann Intern Med 2000;132:391-402.

8 Compendium of strategies to prevent HAls. Available: http://www. sheaonline.org/about/compendium.cfm

9 Ista E, van der Hoven B, Kornelisse RF, et al. Effectiveness of insertion and maintenance bundles to prevent central-lineassociated bloodstream infections in critically ill patients of all ages: a systematic review and meta-analysis. Lancet Infect Dis 2016;16:724-34.

10 Dumyati G, Concannon C, van Wijngaarden E, et al. Sustained reduction of central line-associated bloodstream infections outside the intensive care unit with a multimodal intervention focusing on central line maintenance. Am J Infect Control 2014;42:723-30.

11 Klintworth G, Stafford J, O'Connor M, et al. Beyond the intensive care unit bundle: implementation of a successful hospital-wide initiative to reduce central line-associated bloodstream infections. Am J Infect Control 2014;42:685-7.

12 Resar R, Griffin FA, Haraden C. Using care bundles to improve health care quality: IHI innovation series white paper Institute for Healthcare Improvement; 2012.

13 CDC. Bloodstream infection event (Central line-associated bloodstream infection and non-central line-associated bloodstream infection), 2017. Available: https://www.cdc.gov/nhsn/pdfs/ pscmanual/4psc_clabscurrent.pdf

14 Coopersmith CM, Rebmann TL, Zack JE, et al. Effect of an education program on decreasing catheter-related bloodstream infections in the surgical intensive care unit. Crit Care Med 2002;30:59-64.

15 Coopersmith CM, Zack JE, Ward MR, et al. The impact of bedside behavior on catheter-related bacteremia in the intensive care unit. Arch Surg 2004;139:131-6.

16 Eggimann P, Harbarth S, Constantin MN, et al. Impact of a prevention strategy targeted at vascular-access care on incidence of infections acquired in intensive care. Lancet 2000;355:1864-8.

17 Fakih MG, Jones K, Rey JE, et al. Sustained improvements in peripheral venous catheter care in non-intensive care units: a quasiexperimental controlled study of education and feedback. Infect Control Hosp Epidemiol 2012;33:449-55.

18 Santana SL, Furtado GHC, Wey SB, et al. Impact of an education program on the incidence of central line-associated bloodstream infection in 2 medical-surgical intensive care units in Brazil. Infect Control Hosp Epidemiol 2008;29:1171-3.

19 Shedlarski A, White-Williams C. An evidence-based project to decrease catheter-related bloodstream infections. Nurs Crit Care 2013;8:39-43.

20 Warren DK, Zack JE, Mayfield JL, et al. The effect of an education program on the incidence of central venous catheter-associated bloodstream infection in a medical ICU. Chest 2004;126:1612-8.

21 Gupta P, Shiju S, Chacko G, et al. A quality improvement programme to reduce hospital-acquired pressure injuries. BMJ Open Qual 2020;9:e000905.

22 O'Grady NP, Alexander M, Burns LA, et al. Guidelines for the prevention of intravascular catheter-related infections. Clin Infect Dis 2011;52:e162-93.

23 Levy I, Katz J, Solter E, et al. Chlorhexidine-impregnated dressing for prevention of colonization of central venous catheters in infants and children: a randomized controlled study. Pediatr Infect Dis J 2005;24:676-9.

24 Yébenes JC, Delgado M, Sauca G, et al. Efficacy of three different valve systems of needle-free closed connectors in avoiding access of microorganisms to endovascular catheters after incorrect handling. Crit Care Med 2008;36:2558-61.

25 Smith B. Peripheral intravenous catheter dwell times: a comparison of 3 securement methods for implementation of a 96-hour scheduled change protocol. J Infus Nurs 2006;29:14-17.

26 Ista E, van der Hoven B, Kornelisse RF, et al. Effectiveness of insertion and maintenance bundles to prevent central-lineassociated bloodstream infections in critically ill patients of all ages: a systematic review and meta-analysis. Lancet Infect Dis 2016;16:724-34.

27 Langley GL, Moen R, Nolan KM. The improvement guide: a practical approach to enhancing organizational performance. 2nd edition. San Francisco: Jossey-Bass Publishers, 2009.

28 Pronovost P. Interventions to decrease catheter-related bloodstream infections in the ICU: the keystone intensive care unit project. $A m \mathrm{~J}$ Infect Control 2008;36:S171.e1-S171.e5.

29 Raad II, Hohn DC, Gilbreath BJ, et al. Prevention of central venous catheter-related infections by using maximal sterile barrier precautions during insertion. Infect Control Hosp Epidemiol 1994;15:231-8.

30 Chaiyakunapruk N, Veenstra DL, Lipsky BA, et al. Chlorhexidine compared with povidone-iodine solution for vascular catheter-site care: a meta-analysis. Ann Intern Med 2002;136:792-801.

31 Jain M, Miller L, Belt D, et al. Decline in ICU adverse events, nosocomial infections and cost through a quality improvement initiative focusing on teamwork and culture change. Qual Saf Health Care 2006;15:11:235-9.

32 Richards GA, Brink AJ, Messina AP, et al. Stepwise introduction of the 'Best Care Always' central-line-associated bloodstream infection prevention bundle in a network of South African hospitals. J Hosp Infect 2017;97:86-92.

33 Sax H, Allegranzi B, Uçkay I, et al. 'My five moments for hand hygiene': a user-centred design approach to understand, train, monitor and report hand hygiene. J Hosp Infect 2007;67:9-21. 